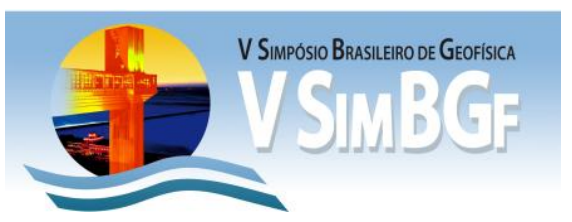

\title{
GPR 3D COMO MÉTODO NÃO INVASIVO PARA ESTUDO DO CRESCIMENTO E BIOMASSA DE RAIIZES DE ÁRVORES DE FLORESTAS TROPICAIS
}

Amanda Almeida Rocha (PHYGEO - Soluções em Geofísica e Geotecnologia - amandarocha@phygeo.com.br)

Frederico R. F. R. de Oliveira e Sousa (PHYGEO-Soluções em Geofísica e Geotecnologia-fredericosousa@phygeo.com.br)

Mônica Giannoccaro Von Huelsen (Universidade de Brasília - monicavon.huelsen@gmail.com)

Welitom Rodrigues Borges (Universidade de Brasília - welitom@unb.br)

Copyright 2012, SBGf - Sociedade Brasileira de Geofísica

Este texto foi preparado para a apresentação no V Simpósio Brasileiro de Geofísica Salvador, 27 a 29 de novembro de 2012. Seu conteúdo foi revisado pelo Comitê Técnico do V SimBGf, mas não necessariamente representa a opinião da SBGf ou de seus associados. É proibida a reprodução total ou parcial deste material para propósitos comerciais sem prévia autorização da SBGf.

\section{Resumo}

O objetivo deste trabalho consiste na estimativa da biomassa das raízes de três espécies de árvores de floresta tropical, através do uso do GPR e da comparação com a metodologia in situ. Os perfis de GPR 2D foram adquiridos pela técnica do afastamento comum com antenas blindadas de alta frequência $(900 \mathrm{MHz})$, uma vez que as principais raízes auxiliares da planta atingem apenas os primeiros $2 \mathrm{~m}$, onde há uma notável supremacia do compartimento solo como grande reservatório de carbono. Os resultados evidenciaram o comportamento e a geometria das raízes ao longo do solo, possibilitando a estimativa do volume com precisão de 2 a $25 \%$ em função da espécie arbórea.

\section{Introdução}

Até a década de 90, os estudos geofísicos atribuíam às raízes de plantas como fontes de ruídos (Doolittle e Miller 1991; Barker e Doolittle, 1992; Silveira et al., 2010). Contudo, trabalhos como o de Hruska et al. (1999 apud Butnor et al., 2001), inovaram nessa área usando o GPR para mapear raízes de carvalhos (maiores que $3 \mathrm{~cm}$ de diâmetro), com mais de 50 anos de idade. Assim, outros geofísicos como Butnor et al. (2001) usando antenas acima de $1 \mathrm{GHz}$ conseguiram detectar raízes de até 0,5 $\mathrm{cm}$ de diâmetro no sudeste do Estados Unidos e Vianden et al. (2010) na Alemanha, aplicaram a técnica para estudar o comportamento de raízes em áreas urbanas. Contudo, no Brasil, engenheiros ambientais se queixam da ausência de técnicas indiretas de estudo de raízes, como exemplo Silva (2007), onde alegou que nos trópicos úmidos há poucos estudos com biomassa de raízes grossas e esses poucos estudos utilizam parcelas fixas para estimar a biomassa, e que ainda não há na literatura modelos alométricos para estimar de forma indireta, a biomassa de raízes grossas de regiões tropicais. Nesse sentido o trabalho proposto por Sousa et al. (2011) estimou com sucesso, a biomassa de um grupo de guapuruvus com 0 GPR até a profundidade de 8 metros.
Este trabalho tem o objetivo de verificar indireta e diretamente o diâmetro de algumas raízes de árvores de florestas tropicais através do uso do GPR e de verificação direta em poços de inspeção.

\section{Metodologia}

No estudo foram selecionadas árvores dentro do campus da Universidade de Brasília (Figura 1). As árvores estudadas compreendem: a Acacia farnesiana (L.) Willd (esponjinha), a Mangifera indica (mangueira) e a Schizolobium parahyba (guapuruvu). Cada espécie possui uma densidade média distinta de madeira, o que corrobora no cálculo individual de carbono para cada tipo de raiz.

A esponjinha é um arbusto da família Leguminosae (Fabaceae) e subfamília das Mimosáceas, pertence à espécie mais abundante do gênero Acacia, típica das savanas africanas, sendo encontrada hoje em climas tropical e subtropical, é resistente ao fogo e não tolera o frio, são usadas para o reflorestamento de terras áridas degradadas devido a sua boa adaptação em ambientes áridos e semiáridos. Possuem valor econômico destacado para madeireiras e forrageiras. Distinguem-se por sua copa plana, seus duríssimos espinhos, suas folhas bipidadas, suas flores de coloração amarela e seus frutos que são vagens pretas. É rica em tanino que se utiliza na elaboração de tintas e produtos farmacêuticos, além do extrato etanólico da planta mostrar uma intensa atividade hipoglicêmica (SILVA, 2011).

A mangueira é uma árvore frondosa da família das Anacardiáceas originária do sul da Ásia, hoje cultivada em todos os países tropicais e subtropicais. Distingue-se pelo seu fruto, a manga, que é altamente nutritivo possuindo vitaminas A, B e C. Atualmente, a mangueira se destaca entre as fruteiras mais exportadas do mundo, estando o Brasil entre os maiores exportadores junto ao México, Filipinas, Paquistão e Índia (FONSECA, 2002). De modo que possui enorme importância econômica para o país, principalmente para a região semiárida, no Vale do São Francisco (EMBRAPA, 2004). Foi consagrada como uma das espécies mais plantadas desde a década de 60 para a arborização urbana do plano piloto de Brasília (LIMA, 2009).

O guapuruvu é uma leguminosa característica da floresta atlântica, mas que também ocorre naturalmente na floresta amazônica. Pesquisas voltadas para avaliação 
de espécies nativas para o reflorestamento na Amazônia indicam que o guapuruvu é uma das espécies nativas que apresenta melhores resultados: após o primeiro ano de cultivo, suas mudas chegam a atingir $1,6 \mathrm{~m}$ (EMBRAPA, 2009).

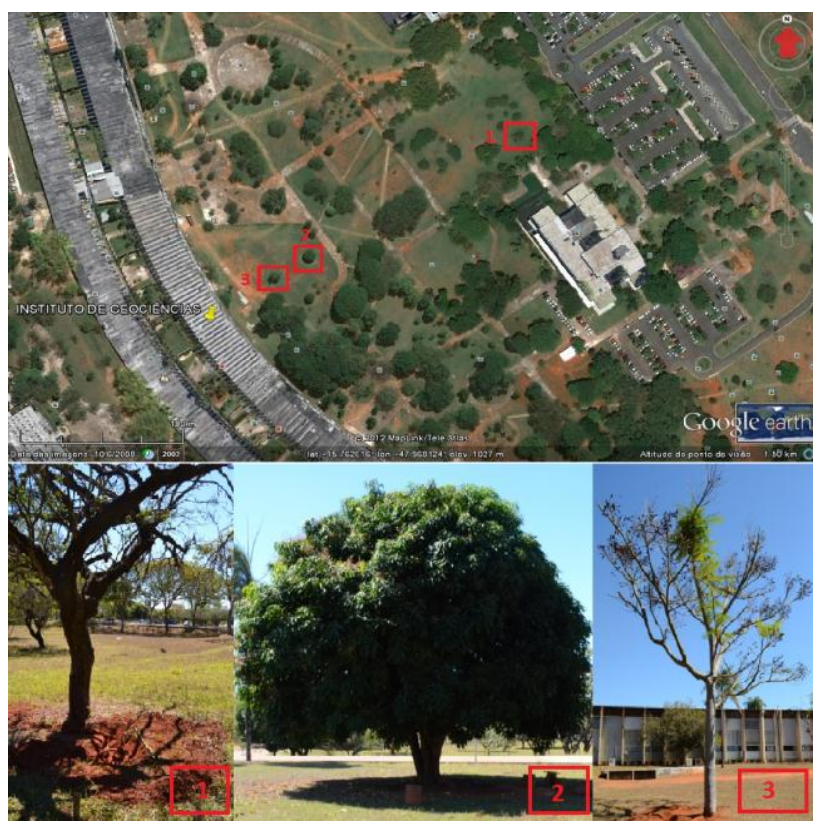

Figura 1 - Localização das árvores estudadas, fonte Google Earth. Fotografias evidenciam as espécies de estudo: 1) Esponjinha; 2) Mangueira e 3) Guapuruvu.

Os vegetais são conhecidos por serem capazes de efetuar trocas de $\mathrm{CO}_{2}$ com a atmosfera pelos processos da fotossíntese e da respiração. A fotossíntese é o processo pelo qual a planta sintetiza compostos orgânicos e libera oxigênio a partir da presença de luz, água e $\mathrm{CO}_{2}$ da atmosfera. Os compostos orgânicos produzidos são carboidratos, como glicose, amido e celulose, que são essenciais para a sobrevivência e o crescimento da planta e o acúmulo de biomassa (IPCC, 2006). De modo que a melhoria da estimativa da biomassa é imprescindível para a consistência na quantificação do carbono fixado nos ecossistemas florestais (BROWN, 1997, BROWN et al., 1989), sendo que a maior parte das pesquisas relativas à biomassa tem focado nas estruturas arbóreas, pois representam a maior fração da biomassa total (BROWN et al. 1992), resultado intimamente relacionado ao Protocolo de Kyoto e ao mercado mundial de carbono, que está em franca ascensão, de acordo com o relatório State Trends of the Carbon Market publicado pelo Banco Mundial em 2011 com valores totais negocias na ordem e US\$ 142 bilhões. Em um ecossistema existem diversas formas de acúmulo de biomassa (MARTINS, 2004), que podem ser considerados em um projeto no Mecanismo de Desenvolvimento Limpo, são cinco os reservatórios: biomassa acima do solo (parte aérea da árvore: tronco, galhos e folhas); biomassa abaixo do solo (raízes das árvores); serrapilheira (camada de resíduos orgânicos depositado no solo da floresta); madeira morta (árvores e arbustos mortos) e carbono no solo (CGEE, 2008).

O carbono fixado pelo reservatório de biomassa viva (acima e abaixo do solo) pode representar entre 70 e $95 \%$ do $\mathrm{CO}_{2}$ sequestrado durante o crescimento de uma floresta tropical (CGEE, 2008). De modo que na prática, segundo as metodologias aprovadas no United Nations Framework Convention on Climate Change (UNFCCC), observa-se que a maioria se restringe ao reservatório de biomassa viva, desconsiderando outros reservatórios.

Existem dois métodos para estimativa de biomassa viva: o método direto e o método indireto. O método direto consiste na derrubada de todas as árvores dentro de uma parcela fixa e pesagem no local do tronco, galhos, folhas e raízes. Amostras de cada componente são coletadas e posteriormente secadas em estufa para determinação do peso seco (Silva, 2007). Para a estimativa da biomassa abaixo do solo, a metodologia típica consiste em abertura de trincheiras, espacialmente distribuídas, para a contabilização de raízes médias e finas, e escavação total para a quantificação de raízes grossas e, da mesma forma que a biomassa acima do solo, aplica-se o método de análises de regressão, para o desenvolvimento de modelos alométricos para estimativa da biomassa de raízes (BROWN, 2002b apud FEREZ, 2010). A estimativa direta da biomassa em floresta tropical é dificultada pelas dimensões das árvores, tornando-se trabalhosa, cara e dependente de tempo e recursos humanos adequados. Além disso, a legislação ambiental brasileira vigente visa proteger os ecossistemas, dificultando a aplicação de métodos destrutivos (BURGER; DELITTI, 1999; VIEIRA, et al. 2008 apud PREISKORN 2011).

Os métodos indiretos envolvem estimativas de biomassa viva através de modelagem matemática (equações alométricas), onde uma ou mais variáveis de fácil obtenção, como diâmetro, altura da árvore ou a densidade, são correlacionadas com a biomassa seca. Outro tipo de método indireto é o uso de técnicas de sensoriamento remoto (SILVEIRA, 2008). Como o acúmulo de biomassa depende do tipo florestal, da distribuição etária da floresta, da composição das espécies, da estrutura e do grau de distúrbio, assim como das características regionais, como o clima, o tipo de solo e o regime pluviométrico (BROWN et al. 1989; MARTINS, 2004), as ferramentas de Sistemas de Informações Geográficas (SIG) e o sensoriamento remoto mostram-se de grande importância, permitindo a análise em larga escala. Contudo, esta técnica permite a estimativa de forma indireta somente da parte da biomassa viva acima do solo, de modo que o método do radar de penetração no solo aparece para corroborar com as metodologias indiretas, com foco no reservatório biomassa abaixo do solo, uma vez que este é o componente mais difícil e demorado de ser quantificado, em qualquer ecossistema florestal, devido às dificuldades operacionais, além de que os métodos geralmente não são padronizados (BRITEZ et al., 2006).

O radar de penetração do solo ou GPR é um método geofísico que emprega ondas eletromagnéticas para localizar estruturas e feições geológicas rasas, consiste 
na emissão e recepção de ondas eletromagnéticas refletidas nas interfaces do meio físico, produzindo como resultado uma imagem de alta resolução da subsuperfície.

Os principais fatores que controlam o sinal do equipamento são: a frequência e a velocidade da onda eletromagnética no meio, o coeficiente de reflexão (contraste de permissividade dielétrica entre os meios) e a atenuação do meio (ANNAN, 1992). Dessa forma, a escolha da frequência central do GPR deve ser sempre precedida da identificação do solo, uma vez que a quantidade de argila, teor de sais ou água podem influenciar diretamente na atenuação do sinal do radar (DOOLITTLE e BUTNOR, 2009).

As principais classes de solo da região do bioma Cerrado são representadas pelos solos do DF (BUOL e CLINE, 1973, apud MARTINS et al., 2008), que possuem três classes mais importantes, denominadas Latossolo Vermelho (LV), Latossolo Vermelho-Amarelo (LVA) e Cambissolo (CX), com representatividade territorial de $85,49 \%$. Somente os Latossolos representam a maior parte da área com $54,47 \%$, divididos em LV $(38,63 \%)$ e LVA $(15,84 \%)$

As amostras estão plantadas na classe LV que ocorre principalmente nos topos das chapadas, principais divisores com topos planos, na depressão do Paranoá e na Bacia do Preto (MARTINS et al. 2008).

Antenas de altas frequências proporcionam melhores resultados em solos secos e eletricamente resistivos, de modo que a atenuação é proporcional à condutividade elétrica do meio, ou seja, quanto maior a condutividade maior a atenuação da onda eletromagnética (ANNAN, 1992). Antenas com frequência entre $900 \mathrm{MHz}$ e 1,5 MHz têm sido utilizadas para algumas investigações rasas e também em solos arenosos. Contudo, as antenas mais utilizadas para investigações de solo têm frequência central entre 100 e $500 \mathrm{MHz}$, pois conseguem atingir uma maior profundidade. Para solos orgânicos, responsáveis por uma elevada atenuação do sinal eletromagnético, onde grandes profundidades de investigação são necessárias à pesquisa, antenas de baixas frequências, entre 70 e $200 \mathrm{MHz}$, são comumente utilizadas. (DOOLITTLE e BUTNOR, 2009). A Tabela 1 mostra a variação de propriedades físicas de alguns solos em função de suas composições.

Tabela 1 - Intervalo da constante dielétrica, condutividade elétrica e permeabilidade magnética (Annan, 1992; Porsani, 1999; Prado, 2000).

\begin{tabular}{c|ccc}
\hline Tipo de solo & $\boldsymbol{K}$ & $\boldsymbol{\mu r}$ & $\boldsymbol{\sigma}_{0}(\mathbf{m} \mathbf{S} / \mathbf{m})$ \\
\hline Solo arenoso & 2,6 & 1 & 0,14 \\
Solo arenoso & 25 & 1 & 6,9 \\
saturado & 2,4 & 1 & 0,27 \\
Solo argiloso & 15 & 1 & 50 \\
Solo argiloso & $3-5$ & 1 & $<0.1$ \\
saturado & Raiz & &
\end{tabular}

De acordo com os valores da Tabela 1, a constante dielétrica representa a característica física particular do meio em que o pulso do radar está percorrendo. Segundo Cunha (2009), a constante dielétrica passa a ter papel de destaque ao se trabalhar com métodos eletromagnéticos de frequências elevadas $(>1 \mathrm{MHz})$. Para frequências entre $1 \mathrm{MHz}$ e $2 \mathrm{GHz}$, o conteúdo em água governa as propriedades dielétricas dos materiais, uma vez que o valor da permissividade relativa da água é dezenas de vezes maior que a permissividade característica das raízes. Dessa forma o equipamento geofísico GPR é capaz de caracterizar o meio estudado como latossolo vermelho devido à sua constante dielétrica característica, bem como localizar a posição e dimensões das raízes das árvores de forma não invasiva (SOUSA et al. 2011).

$\mathrm{Na}$ aquisição de dados foi utilizado o GPR modelo SIR3000 (fabricado pela empresa Geophysical Survey Systems, Inc.) acoplado a um par de antenas blindadas com frequência central de $900 \mathrm{MHz}$ e uma roda odométrica (Figura 2). Os dados foram adquiridos através da técnica do afastamento comum, onde um par de antenas (uma transmissora e outra receptora) é deslocado ao mesmo tempo ao longo de cada perfil, permitindo uma visualização em tempo real dos refletores no subsolo. Os dados foram coletados ao longo de perfis de $4 \mathrm{~m}$, equiespaçados em $5 \mathrm{~cm}$, onde as três espécies ficaram no centro de um área de $16 \mathrm{~m}^{2}$, para cada um dos levantamentos.

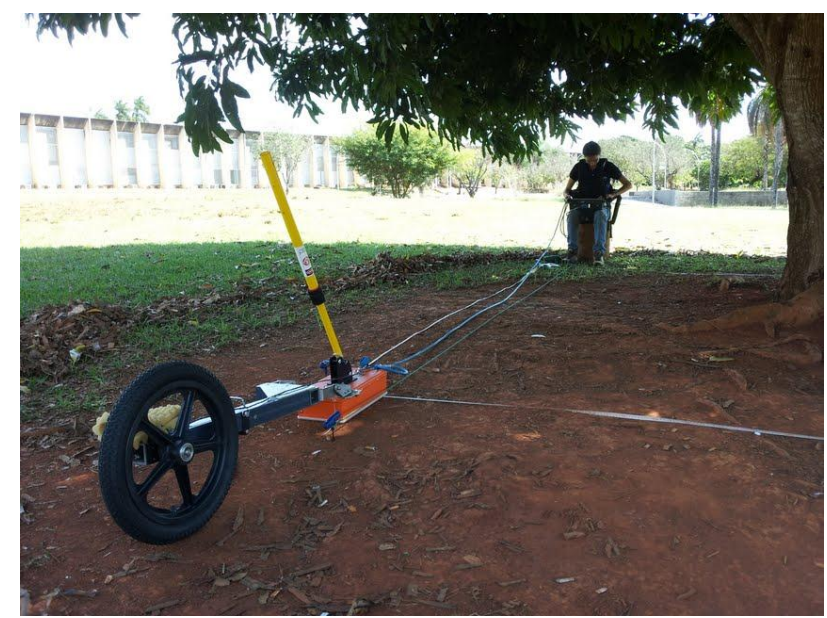

Figura 2: Foto evidencia o sistema GPR utilizado na aquisição de dados.

Após a etapa de aquisição, os dados foram processados no software ReflexW, versão 6.0 (SANDMEIER, 2011). As etapas da rotina de processamento foram aplicadas de acordo com as características dos dados e dependem, fundamentalmente, do intérprete (BORGES, 2010). O fluxo do processamento consistiu em conversão do formato do arquivo, ajuste do tempo zero, remoção de background, ganho (declipping), migração fk, conversão do tempo para profundidade (velocidade de ajuste hiperbólico encontrada nas raízes - 0,12m/ns), e 
conversão dos dados de amplitude verdadeira para envelope do traço.

Os perfis 2D de cada linha de aquisição foram interpretados com base nos padrões geométricos de reflexão do pulso eletromagnético e possibilitam uma boa estimativa dos diâmetros das raízes das espécies, assim como o volume das mesmas através do envelope do traço. Com a interpolação dos resultados obtidos nos perfis 2D, foi possível a elaboração de mapas 3D do padrão do sistema de raízes de cada espécie estudada.

\section{Resultados}

Os resultados obtidos com as antenas blindadas de 900 $\mathrm{MHz}$ possibilitam a identificação de difrações hiperbólicas relacionadas às raízes de árvores, com o ajuste da penetração do sinal até a profundidade máxima de $2 \mathrm{~m}$. Foram gerados depth slices nos blocos de GPR 3D das três diferentes espécies, o que possibilitou uma visualização do padrão do crescimento lateralizado das raízes e posterior comparação com a metodologia in situ, onde ficou evidente a boa precisão do GPR para o estudo de caso.

A Figura 3 exemplifica algumas difrações hiperbólicas relacionadas às raízes da esponjinha, assim como a variação do diâmetro das mesmas com o envelope do traço. Comparando-se o resultado do envelope do traço (Figura 3b) com a amostragem convencional (Figura 3c) observa-se que houve um erro de $25 \%$ nesta espécie de árvore. Todavia o GPR mostra-se efetivo para mapeamento da distribuição lateral das raízes grossas (Figura 3d).

A Figura 4 exemplifica algumas difrações hiperbólicas relacionadas às raízes da mangueira, assim como a variação do diâmetro das mesmas com o envelope do traço. Comparando-se o resultado do envelope do traço (Figura 4b) com a amostragem convencional (Figura 4c) observa-se que houve um erro de $12 \%$ nesta espécie de árvore. Todavia o GPR mostra-se efetivo para mapeamento da distribuição lateral das raízes grossas (Figura 4d).

A Figura 5 exemplifica algumas difrações hiperbólicas relacionadas às raízes do guapuruvu, assim como a variação do diâmetro das mesmas com o envelope do traço. Comparando-se o resultado do envelope do traço (Figura 5b) com a amostragem convencional (Figura 5c) observa-se que houve um erro de $2 \%$ nesta espécie de árvore. Além dos bons ajustes entre os métodos, o GPR mostra-se efetivo para mapeamento da distribuição lateral das raízes grossas (Figura $5 \mathbf{d}$ ).

A quantidade de biomassa em uma floresta é o resultado da diferença da produção da fotossíntese e o consumo pela respiração, que é comumente expressa como peso seco por unidade de área (BROWN, 1997).

Para a estimativa do total de biomassa das raízes, se obteve os valores do volume de raiz para cada espécie, encontrado com o GPR, correlacionado aos valores de densidades média da madeira e ao teor de água.
Para o gênero Acácia a densidade básica média da madeira varia entre 0,488 a $0,7 \mathrm{~g} / \mathrm{cm}^{3}$ (ALENCAR, 2009), para a mangueira a densidade média varia 0,52 a 0,7 $\mathrm{g} / \mathrm{cm}^{3}$ (RICHTER et al. 2009), e a densidade média usada para a madeira do guapuruvu é $0,32 \mathrm{~g} / \mathrm{cm}^{3}$ (LORENZI, 2002).

Segundo Silva (2007), o teor de água ponderado por partes de uma árvore, incluindo suas raízes, é de 41,6\%, com incerteza de $2,8 \%$. Portanto, subtraindo a contribuição da água no total da massa, encontramos o valor total da biomassa das raízes de cada espécie.

A estimativa do estoque de carbono da vegetação foi obtida pelo produto da biomassa florestal e a concentração de carbono. A concentração de carbono na biomassa de florestas tropicais está entre 46 e $52 \%$ (HIGUCHI et al., 2004, IPCC, 2006). Contudo, muitos autores consideram $50 \%$ de carbono na biomassa (BROWN, GILLSEPIE, LUGO 1989; BROWN, 1997; BRITEZ et al., 2006).

Em função das estimativas de diâmetro de raízes encontrados com o GPR, pode-se concluir que o volume de biomassa e consequente carbono sequestrado, estimados para a esponjinha tem precisão de $75 \%$, para a mangueira de $88 \%$ e para o guapuruvu de $98 \%$.

\section{Discussão e Conclusões}

Os resultados obtidos mostram que o GPR é um bom método para estimar, de forma indireta, o crescimento, dinâmica e biomassa das raízes grossas de algumas árvores típicas das florestas tropicais.

As principais vantagens do GPR, quando comparado às técnicas tradicionais de estimativa de biomassa, advêm da redução de custos, precisão das medidas e realização do trabalho de campo em curto tempo. É importante ressaltar que o GPR é uma técnica geofísica de impacto ambiental mínimo.

Este estudo será aprofundado com a melhoria das estimativas da densidade das raízes, assim como do teor de água em cada espécie, etapa crucial para a consolidação do método GPR neste tipo de levantamento ambiental.

\footnotetext{
Agradecimentos

Agradecemos primeiramente a equipe PHYGEO Soluções em Geofísica e Geotecnologia pela viabilização do trabalho e aquisição dos dados. Somos gratos ao Laboratório de Geofísica Aplicada do Instituto de Geociências da Universidade de Brasília, pela cessão dos equipamentos e apoio logístico para 0 processamento dos dados. Também agradecemos aos profissionais do Observatório Sismológico e aos profissionais da Jardinagem da UnB pelo apoio.
} 

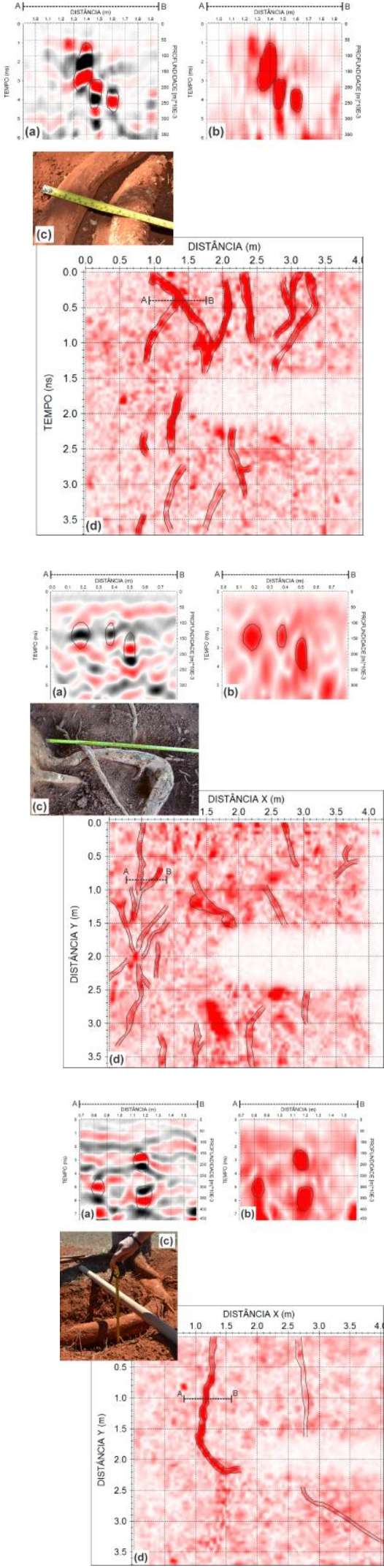

Figura 3 - Resultados de GPR obtidos para a árvore Esponjinha. (a) seção de amplitude verdadeira com refletores relacionados a raízes. (b) seção de envelope do traço com as raízes interpretadas. (c) Foto evidencia as raízes encontradas nas seções anteriores. (d) Depth Slice ressaltando algumas raízes verificadas na profundidade de $0,15 \mathrm{~m}$.

Figura 4 - Resultados de GPR obtidos para a árvore Mangueira. (a) seção de amplitude verdadeira com refletores relacionados a raízes. (b) seção de envelope do traço com as raízes interpretadas. (c) Foto evidencia as raízes encontradas nas seções anteriores. (d) Depth Slice ressaltando algumas raízes verificadas na profundidade de $0,08 \mathrm{~m}$.

Figura 5 - Resultados de GPR obtidos para a árvore Guapuruvu. (a) seção de amplitude verdadeira evidenciando refletores relacionados a raízes. (b) seção de envelope do traço com as raízes interpretadas. (c) Foto mostra a raiz encontrada nas seções anteriores. (d) Depth Slice ressaltando algumas raízes verificadas na profundidade de $0,17 \mathrm{~m}$. 


\section{Referências}

ALENCAR, G.S.B. Qualidade da madeira de espécies do gênero Acacia plantadas no Brasil. Tese (Doutorado). Escola Superior de Agricultura Luiz Queiroz, Piracicaba/SP 2009

ANNAN, A.P., 1992. Ground penetration radar workshop notes. Sensors; Software, Inc., Internal Report, 130p.

BORGES, W.R.; BLUM, M.L.B.; BRANCO, R.M.C.; SILVA, L.M.C.; PORSANI, M.J.; BRICHTA, A.; AMORIM, A.N.; PEDROSA, J.R.; SANTOS, V.R.N.; ALBUQUERQUE, J.; MACEDO, P.B.; SANTOS, E.C.S.; BRASIL, D.L: MACHADO, M.A.; MARTINS, J.A.; MELLO, G.A. Aplicação da Geofísica Forense na Busca de Vestígios de Pessoas Desaparecidas na Guerrilha do Araguaia. In: IV Simpósio Brasileiro de Geofísica, Brasília-DF, 2010.

BORGES, W.R. Investigações geofísicas na borda da bacia sedimentar de São Paulo, utilizando-se GPR e eletrorresistividade. Dissertacão (Mestrado em Geofísica) Departamento de Geofísica, IAG/USP, São Paulo, 2002

BRITEZ, R.M. de; BORGO, M.; TIEPOLO, G.; FERRETTI, A.; CALMON M.; HIGA, R. Estoque e incremento de carbono em florestas povoamentos de espécies arbóreas com ênfase na Floresta Atlântica do Sul do Brasil. Colombo: Embrapa Florestas; Curitiba: Sociedade de Pesquisa em Vida Selvagem e Educação Ambiental, 2006. 165p.

BROWN, S. Estimating Biomass and Biomass Change of Tropical Forests: a primer. FAO Forestry Paper 134. Rome; 1997.

BROWN, S.; GILLESPIE, A.J.R.; LUGO, A.E. Biomass estimation methods for tropical forests with applications to forest inventory data. Forest Science, Maryland, v. 35, n.4, p. 881-902, Dec. 1989.

BROWN, S.; LUGO, A.E. Aboveground biomass estimates for tropical moist forests of the Brazilian Amazon. Interciencia, Caracas, v. 17, n. 1, p. 8-18, Feb. 1992.

BUTNOR, J.R.; DOOLITTLE, J.A.; KRESS, L.; COHEN, S.; JOHNSEN, K.H. 2001. Use of ground-penetrating radar to study tree roots in the southeastern United States. In: Tree Physiology. Victoria, Canada. Vol. 21: p. $1269-1278$

CENTRO DE GESTÃO E ESTUDOS ESTRATÉGICOS. Manual de Capacitação sobre Mudança do Clima e Projetos de Mecanismo de Desenvolvimento Limpo (MDL). Brasília, 2008. 276p.

CUNHA, L. F. J. Métodos elétricos e eletromagnéticos na avaliação da contaminação de aquíferos por atividades de mineração e indústria de curtume. 2009. 131f. Tese (Doutorado em Geofísica) - Instituto de Geofísica, Universidade Federal da Bahia, Salvador. 2009.

DOOLITTLE, J.A. e BUTNOR, J.R. Ground Penetrating Radar: Theory and applications. Chapter 6: Soils, peatlands, and biomonitoring. Editor Harry M. Jol. Elsevier Science 2009.

$\begin{array}{llll}\text { EMBRAPA - CARVALHO, Paulo Ernani Ramalho. Guapuruvu. Circular } \\ \text { Técnica } 104, \quad \text { ano } 2005 . & \text { Disponível } & \text { em: }\end{array}$ http://www.cnpf.embrapa.br/publica/circtec/edicoes.htm . Acesso: 20/08/2012.

EMBRAPA - MEDEIROS, Daniel. Pesquisa Avalia Espécies Nativas para Recuperação Ambiental na Amazônia 27/01/2009. Disponível em: http://www.embrapa.br/imprensa/noticias/2009/janeiro/4a-

semana/pesquisa-avalia-especies-nativas-para-recuperacao-ambientalna-amazonia. Acesso: 28/03/2011.

EMBRAPA - SILVA, P.C.G., CORREIA, R.C., Cultivo da Mangueira. Socioeconomia. A importância econômica e social da mangueira na região semi-árida. Versão eletrônica Julho/2004. Disponível em: http://sistemasdeproducao.cnptia.embrapa.br/FontesHTML/Manga/Cultivo daMangueira/socioeconomia.htm. Acesso: 23/06/2012.

FEREZ, A.P.C. Efeito de práticas silviculturais sobre as taxas iniciais de sequestro de carbono em plantios de restauração da Mata Atlântica. 108p. Dissertação (Mestrado) - USP - Escola Superior de Agricultura "Luiz de Queiroz". Piracicaba, 2011.

FONSECA, Nelson. Paclobutrazol e estresse hídrico no florescimento e produção da mangueira (Mangifera indica L.) "Tommy Atkins". Lavras: UFLA, 2002.
HIGUCHI, N.; CHAMBERS, J.; SANTOS, J.; RIBEIRO, R.J.; PINTO, A.C.M.; SILVA, R.P.; ROCHA, R.M.; TRIBUZY,E.S. Dinâmica e balanço do carbono da vegetação primária da Amazônia Central. Floresta, Curitiba, v. 34, n. 3, p. 295-304, set/dez 2004.

IPCC Guidelines for National Greenhouse Gas Inventories (GNGGI), 2006. Disponível em: http://www.ipccnggip.iges.or.ip/public/2006gl/index.html . Acesso: 10/08/2012.

LIMA, R. M. C. Avaliação da arborização urbana do plano piloto. Dissertação de Mestrado em Ciências Florestais, Publicação PPGRFL.DM - 117/2009, departamento de Engenharia Florestal, Universidade de Brasília, Brasília, DF, 85p.

LORENZI, H. Árvores brasileiras: Manual de Identificação e cultivo de plantas arbóreas nativas do Brasil. Nova Odessa. Ed. Plantarum. V.01 368p. 2002.

KOSSOY, A: AMBROSI, P. State and Trends of the Carbon Market World Bank Institute - WBI, 2011, p. 1-2.

MARTINS, E.S.; JUNIOR, O.A.C.; MELLO, G.A.; REATTO, A.; MORAES, R.A.V.; PIRES, A.C.B.; GUIMARAES, R.F.. Mapeamento da Superfície Basal do Manto de Intemperismo pelo Comportamento da Resistividade Elétrica do Distrito Federal. In: Revista Brasileira de Geomorfologia, 2008, vol. 9, no 2: p. $15-28$.

MARTINS, O.S. Determinação do potencial de sequestro de carbono na recuperação de matas ciliares na região de São Carlos - SP, 2004. 136p. Tese (Doutorado em Ecologia e Recursos Naturais) - Universidade Federal de São Carlos, São Carlos, 2004.

PORSANI, J.L. Caracterização Geofísica de Alvos Rasos com Aplicações no Planejamento Urbano, Meio Ambiente e Arqueologia: Estudo Sobre o Sítio Controlado do IAG/USP. Relatório de Pesquisa (Processo Fapesp n.ำ 99/12217-5), São Paulo, SP, 84p.

PRADO, R.L. 2000. A sísmica de reflexão e o radar de penetração no solo na investigação geológico-geotécnica em ambientes urbanos: Um estudo na cidade de São Paulo - SP, Brasil. Tese de Doutorado, Instituto de Geociências e Ciências Exatas, UNESP, Campus de Rio Claro - SP $174 p$.

PREISKORN, G.M. Composição florística, estrutura e quantificação do estoque de carbono em florestas restauradas com idades diferentes. 130p. Dissertação (Mestrado) - USP - Escola Superior de Agricultura "Luiz de Queiroz". Piracicaba, 2011.

RICHTER, H.G and DALLWITZ, M.J. 2000 onwards Commercia timbers: descriptions, illustrations, identification, and information retrieval. In English, French, German, Portuguese and Spanish. Version: 25th June 2009. Instituto de Botânica da Academia Chinesa de Ciências. Disponível em http://delta-intkey.com . Acesso em 02/07/2012.

SANDMEIER, K.J. 2011 REFLEXW. Version 6.0. WindowsTM $9 \times / N T / 2000 / X P / 7$ - program for the processing of seismic, acoustic or eletromagnetic reflection, refraction and transmission data. Manual. 516p.

SILVA, C.C.; Araújo, R.O.; Senna, K.X.F.R.; Albuquerque, J.F.C. (UFPe) Determinação da atividade antimicrobiana das cascas do caule de Acácia farnesiana (L.) WILLD. (Leguminosae). In: $51^{\circ}$ Congresso Brasileiro de Química, São Luís/MA, 2011.

SILVA, R.P. Alometria, Estoque e Dinâmica da Biomassa de Florestas Primárias e Secundárias na Região de Manaus (AM). Tese (Doutorado em Ciências de Florestas Tropicais) INPA/UFAM, 2007, p. 11, 27.

SILVEIRA, E. S. F.; SILVA, L. M. C.; NOGUEIRA, G.; LEÃO, D. Investigação Preliminar com os Métodos de Urnas Funerárias do Sitio Nossa Sra. do Perpétuo Socorro (Pará). In: IV Simpósio Brasileiro de Geofísica, Brasília-DF, 2010.

SILVEIRA, P · KOEHLER,H S : SANQUETTA, C R - ARCE, JE O estado da arte na estimativa de biomassa e carbono em formações florestais. Floresta, Curitiba, v. 3, n. 1, p. 185-206. 2008.

SOUSA, F.R.F.R.O; ROCHA, A.A.; BORGES,W.R. Use of ground penetrating radar as a non-invasive method to study the growth, dynamic and biomass contained in the roots of typical trees of tropical rainforest. In: 12TH International Congress of the Brazilian Geophysical Society, 2011. 